\title{
Synovial Membrane-derived Mesenchymal Progenitor Cells From Osteoarthritic Joints in Dogs Possess Lower Chondrogenic-, and Higher Osteogenic Capacity Compared to Normal Joints
}

\section{Michelle Teunissen ( $\nabla$ m.teunissen@uu.nl)}

Utrecht University Faculty of Veterinary Medicine: Universiteit Utrecht Faculteit Diergeneeskunde https://orcid.org/0000-0003-0119-3257

\section{Nora Ahrens}

Utrecht University Faculty of Veterinary Medicine: Universiteit Utrecht Faculteit Diergeneeskunde Lianne Snel

Utrecht University Faculty of Veterinary Medicine: Universiteit Utrecht Faculteit Diergeneeskunde Roberto Narcisi

Erasmus Medical Centre: Erasmus MC

\section{Amir Kamali}

Utrecht University Faculty of Veterinary Medicine: Universiteit Utrecht Faculteit Diergeneeskunde

Gerjo van Osch

Erasmus Medical Centre: Erasmus MC

\section{Björn Meij}

Utrecht University Faculty of Veterinary Medicine: Universiteit Utrecht Faculteit Diergeneeskunde

\section{Simon Mastbergen}

UMC Utrecht: Universitair Medisch Centrum Utrecht

\section{Kavitha Sivasubramaniyan}

Erasmus Medical Centre: Erasmus MC

\section{Marianna Tryfonidou}

Utrecht University Faculty of Veterinary Medicine: Universiteit Utrecht Faculteit Diergeneeskunde

\section{Research Article}

Keywords: synovial membrane, mesenchymal progenitor cells, flow cytometry, immunohistochemistry, trilineage differentiation, CD271, CD34

Posted Date: February 11th, 2022

DOI: https://doi.org/10.21203/rs.3.rs-1335575/v1 
License: (c) (i) This work is licensed under a Creative Commons Attribution 4.0 International License. Read Full License 


\section{Abstract}

Background Synovial membrane-derived mesenchymal progenitor cells (SM-MPCs) are a promising candidate for the cell-based treatment of osteoarthritis (OA) considering their in vitro and in vivo capacity for cartilage repair. However, the OA environment may adversely impact their regenerative capacity. There are no studies for canine (c)SM-MPCs that compare normal to OA SM-MPCs, even though dogs are considered a relevant animal model for OA. Therefore, this study compared cSM-MPCs from normal and OA synovial membrane tissue to elucidate the effect of the OA environment on MPC numbers, indicated by $\mathrm{CD}$ marker profile and colony-forming unit (CFU) capacity, and the impact of the OA niche on trilineage differentiation.

Methods Normal and OA synovial membrane were collected from the knee joints of healthy dogs and dogs with rupture of the cruciate ligaments. The synovium was assessed by histopathological OARSI scoring and by RT-qPCR for inflammation/synovitis-related markers. Presence of cSM-MPCs in the native tissue was further characterized with flow cytometry, RT-qPCR and immunohistochemistry, using the MPC markers; CD90, CD73, CD44, CD271, and CD34. Furthermore, cells isolated upon enzymatic digestion were characterized by CFU capacity, and a population doublings assay. CSM-MPCs were selected based on plastic adherence, expanded to passage 2 and evaluated for the expression of MPC-related surface markers and tri-lineage differentiation capacity.

Results Synovial tissue collected from the OA joints had a significantly higher OARSI score compared to normal joints, and significantly upregulated inflammation/synovitis markers S100A8/9, IL6, IL8 and CCL2. Both normal and OA synovial membrane contained cells displaying MPC properties, including a fibroblast-like morphology, CFU capacity, and maintained MPC marker expression over time during expansion. However, OA cSM-MPCs were unable to differentiate towards the chondrogenic lineage and had low adipogenic capacity in contrast to normal CSM-MPCs, whereas they possessed a higher osteogenic capacity. Furthermore, the OA synovial membrane contained significantly lower percentages of CD90+, CD44+, CD34+ and CD271+ cells.

Conclusions The OA environment has adverse effects on the regenerative potential of CSM-MPCs, corroborated by decreased CFU, population doubling and chondrogenic capacity compared to normal cSM-MPCs. OA cSM-MPCs may be a less optimal candidate for the cell-based treatment of OA than normal cSM-MPCs.

\section{Background}

Mesenchymal progenitor cells (MPCs), also referred to as mesenchymal stem/stromal cells (MSCs), are heterogenous cell populations [1] residing in most adult tissues, including the synovial membrane [2, 3]. Transgenic animal models in combination with tissue injury models have demonstrated that synovial membrane-derived MPCs (SM-MPCs) contribute to joint development and tissue repair upon injury [4]. 
Therefore, SM-MPCs have been considered a promising cell-based treatment strategy for joint diseases such as osteoarthritis (OA) [5].

Under physiological conditions, MPCs participate in tissue homeostasis, remodeling, and repair by ensuring replacement of mature cells that are lost during physiological turnover, senescence, injury, or disease [6]. However, in the osteoarthritic joint, the delicate balance between anabolism and catabolism shifts toward a degenerative environment, characterized by the presence of inflammatory and catabolic mediators [7]. Due to these circumstances, and the poor healing capacity of the cartilage itself, the endogenous repair mechanisms become insufficient $[8,9]$. This catabolic environment also influences the MPC populations in the joint. For example, bone marrow derived MPCs from patients with hip OA, possess a lower proliferation capacity and decreased chondrogenic differentiation capacity [10]. These findings, in addition to an increased senescence, were also reported for SM-MPCs from OA patients [11]. Furthermore, multiple studies show that the number of cells in the synovial membrane expressing MPC markers increases during OA progression [12-14] and their localization changes [12,13]. However, as the exact origin, function and phenotype of MPCs in vivo remain elusive, additional research to elucidate the effect of OA on SM-MPCs and, in turn, their role during OA is necessary.

At this moment, the dog is considered the most relevant animal model for OA due to its translational values in terms of anatomic similarity, disease progression, and translation of outcomes to humans [15, 16]. Importantly, both natural occurring $O A$ as well as surgically induced dog models exist [16, 17], and as such, treatment strategies can be evaluated early in product development for the target species [18]. The presence of SM-MPC has been demonstrated in the normal [19,20] and OA [21] synovial membrane of dogs. However, a direct comparison between normal and OA canine SM-MPCs is lacking, hampering translational studies and advances in this field employing the dog as a model.

In this study, the characteristics of canine (c)SM-MPCs from normal and clinical OA joints were compared. cSM-MSCs of normal joints had higher chondrogenic and adipogenic capacity than cSMMPCs from OA joints, while the latter showed a higher osteogenic capacity. Therefore, differences in the native cMPC population in the synovial membrane were investigated using flow cytometry, RT-qPCR and immunohistochemistry to investigate the expression profile of common MPC/MSC markers CD90, CD44, CD73, CD271, and CD34.

\section{Methods}

\section{Terminology}

In this manuscript, native, uncultured cells derived from the synovial membrane are referred to as mesenchymal progenitor cells (cSM-MPCs). Expanded cells, selected based on plastic-adherence, are referred to as mesenchymal stromal cells (cSM-MSCs) (Supplementary Figure 1)

\section{Animal Samples}


Synovial membrane (SM) was collected from the clinically normal knee joints of skeletally mature, mixedbreed dogs (Supplementary Table 1, $\mathrm{n}=28,18 \pm 7$ months of age, $25 \pm 2 \mathrm{~kg}$ ), euthanized in unrelated experiments conducted in accordance with the guidelines set by the National Central Committee for Animal Experiments (AVD \#115002016531). SM from OA knee joints was collected, with the owner's consent, during standard-of-care surgery (Supplementary Table 1; $n=22,58 \pm 37$ months of age, $38 \pm 12$ $\mathrm{kg}$ ) for cranial cruciate ligament rupture of client-owned dogs at the academic hospital of the Faculty of Veterinary Medicine of Utrecht University. All dogs suffered from secondary knee OA with accompanying joint effusion and increase of intra-articular soft tissue density as confirmed by radiography.

SM tissue samples were processed accordingly for histopathological examination, gene expression profiling, and cell culture experiments. For this purpose, SM was respectively (a) fixed in $4 \%$ neutral buffered formalin (NBF, Klinipath B.V., Duiven, the Netherlands) for paraffin-embedding and (immuno-) histochemical analysis, (b) snap frozen and stored at $-80^{\circ} \mathrm{C}$ for RNA isolation, and (c) stored in aMEM (22561021, Gibco ${ }^{\text {TM }}$, Thermo Fisher Scientific, Waltham, USA) supplemented with 1\% ITS+ Premix (354352, Corning Life Sciences, Amsterdam, The Netherlands), and 1\% penicillin/streptomycin ( $\mathrm{p} / \mathrm{s}$; $10.000 \mathrm{U} / \mathrm{mL}, 15140122$, Gibco ${ }^{\text {TM }}$ ) for a maximum of 24 hours until tissue digestion.

\section{Histopathological evaluation of the SM}

To determine the OA status of the SM, $5 \mu \mathrm{m}$ sections were stained with Haematoxylin/Eosin (HE) (Mayers haematoxylin (109249, Merck), 0.2\% Eosin (115935, Merck)), randomized and scored blindly according to the Osteoarthritis Research Society International (OARSI) canine scoring system [16] by three observers (MT, LS, and SCM).

\section{Synovial membrane tissue digestion and isolation of CSM- MPCs from the synovial membrane}

SM tissue samples were minced, and enzymatically digested using $2 \mathrm{mg} / \mathrm{mL}$ collagenase IV (C5138, Sigma-Aldrich) and $0.08 \mathrm{mg} / \mathrm{mL}$ dispase II (17105041, Gibco ${ }^{\mathrm{TM}}$ ) in Hanks' Balanced Salt Solution (HBSS; $\left.14025050, \mathrm{Gibco}^{\mathrm{TM}}\right)$, at $37^{\circ} \mathrm{C}$ on a shaker for 2-3 hours. Thereafter, remaining undigested tissue was removed by passing the digest through an $18 \mathrm{G}$ needle, and subsequently through $100 \mu \mathrm{m}(542000$, Greiner Bio-One, Alphen aan den Rijn, The Netherlands) and 40 mm (542040, Greiner Bio-One) cell strainers, respectively. The acquired cell suspensions were centrifuged for 8 minutes at $290 \mathrm{~g}$ and washed twice with aMEM containing $10 \%$ fetal bovine serum (FBS; 16000044 , Gibco ${ }^{\mathrm{TM}}$ ) and $1 \% \mathrm{p} / \mathrm{s}$. The total and live cell numbers were determined with a TC20 ${ }^{\text {TM }}$ Automated Cell Counter (145-0101, Bio-Rad).

\section{Colony forming unit capacity of cSM-MPCs}

To evaluate the colony forming unit (CFU) capacity, cSM-MPCs of normal $(n=8)$ and OA $(n=12)$ donors were plated in $58 \mathrm{~cm}^{2}$ Petri dishes (664160, CELLSTAR ${ }^{\circledR}$, Greiner Bio-One) at three cell densities, i.e., \pm 0.25 - 0.5 - and $1 \times 10^{3}$ cells/Petri dish (equivalent to \pm 4 -, 8- and 17 cells $/ \mathrm{cm}^{2}$ ). After 10-14 days under normal culture conditions, cells were stained with $0.5 \%$ crystal violet (C0775, Sigma-Aldrich) in $100 \%$ methanol (MC1060092511, Merck Millipore) for 30 minutes. Colonies containing > 50 cells were counted 
in the appropriate plating density, i.e., the density wherein the individual colonies were not overlapping, and displayed as the percentage of the total seeded cell number.

\section{Expansion of CSM-MSCs}

Following isolation, cells were plated at a density of $\pm 3.3 \times 10^{3}$ cells $/ \mathrm{cm}^{2}$ in T75 culture flasks $(658175$, CELLSTAR®, Greiner Bio-One), hereafter called cSM-MSCs. cSM-MSCs were cultured in humidified, normoxic conditions $\left(5 \% \mathrm{CO}_{2} / 21 \% \mathrm{O}_{2}\right.$ ) at $37^{\circ} \mathrm{C}$, normal culture conditions, in expansion medium (aMEM, $10 \%$ FBS, 1\% p/s, 0.1 mM ascorbic acid 2-phosphate (AsAP; A8960, Sigma-Aldrich, Saint Louis, USA), $1.25 \mu \mathrm{g} / \mathrm{mL}$ Fungizone (Amphotericin B, 15290018, Gibco ${ }^{\text {TM }}$ ) and $1 \mathrm{ng} / \mathrm{mL}$ recombinant human basic fibroblast growth factor-2 (bFGF-2; PHP105, Bio-Rad)). After $\pm 80 \%$ confluency was reached (normal: 6.9 \pm 1.5 days; $O A$ : $9.4 \pm 2$ days) in passage (P) PO, CSM-MSCs were aliquoted and cryopreserved in aMEM with $20 \%$ FBS and $20 \%$ dimethyl sulfoxide (102950, Merck Millipore, Burlington, USA) at $-196^{\circ} \mathrm{C}$ until further analysis.

\section{Population doubling assay of cSM-MSCs until passage 10}

Normal $(n=6)$ and OA $(n=6)$ cSM-MSCs were seeded in triplicate at a density of $\pm 6 \times 10^{3} \mathrm{cells} / \mathrm{cm}^{2}$ in 6 well plates (657160, CELLSTAR®, Greiner Bio-one) under normal culture conditions in expansion medium. Cells were passaged every 3-4 days up to P10. Population doublings per passage was calculated with the following formula: $\mathrm{PD}=\log (\mathrm{Nf})-\log (\mathrm{Ni}) / \log (2)$, where $\mathrm{PD}$ stands for the number of cell divisions in each passage, $\mathrm{Nf}$ for the cell number on the day of passaging and $\mathrm{Ni}$ for the initial seeding number of cells $(6 \times$ $10^{3}$ cells $/ \mathrm{cm}^{2}$ ).

\section{Senescence assay of P0 to P10 cSM-MSCs}

As senescence is thought to play a significant role in OA, it was hypothesized that cSM-MSCs derived from OA joints might undergo senescence earlier compared to normal cSM-MSCs. Therefore, a senescence assay was performed in which normal $(n=6)$ and OA $(n=6)$ cSM-MSCs were seeded at a density of $2,5 \times 10^{4}$ cells $/ \mathrm{cm}^{2}$ in duplicate in chamber slides (PEZGS018, Millipore). After 24 hours $\left(37^{\circ} \mathrm{C}\right.$, $5 \% \mathrm{CO}_{2} / 21 \% \mathrm{O}_{2}$ ), cells were fixed with $4 \% \mathrm{NBF}$ and stained overnight at $37^{\circ} \mathrm{C}$ in the dark with 5 -bromo-4chloro-3-indolyl-ß-D-galactoside (X-gal (B4252, Sigma-Aldrich), $1 \mathrm{mg} / \mathrm{ml}$, in a solution of $40 \mathrm{mM}$ citric acid/sodium phosphate, $5 \mathrm{mM}$ potassium hexacyano-ferrate (II) trihydrate, $5 \mathrm{mM}$ potassium hexacyanoferrate (III), $150 \mathrm{mM}$ sodium chloride and $2 \mathrm{mM}$ magnesium chloride $\left(\mathrm{MgCl}_{2}\right)$ in distilled water $(\mathrm{pH} 6.0)$ ). To visualize nuclei, a counterstain with DAPI (62248, ThermoFisher) was performed. Sections were mounted with FluorSave (345789, VWR), and imaged using Olympus BX43 microscope (Olympus, Tokyo, Japan). Analysis of the percentage of senescent cells was performed with ImageJ (version 1.48) using the Senescence Counter [22], which uses the amount of DAPI positive nuclei to determine the amount of X-gal positive cells.

\section{Tri-lineage differentiation assay of P2 cSM-MSCs}


Tri-lineage differentiation of the cSM-MSCs was performed in P2 as per methods described previously [23], with some small adaptations. For chondrogenic differentiation, normal $(n=7)$, and OA $(n=8)$ P2 cSM-MSCs were cultured in pellets of 100.000 cells/well in a 96-well plate (Corning ${ }^{\circledR}$ Costar $\left.{ }^{\circledR} 7007\right)$ in chondrogenic differentiation medium (DMEM high glucose (31966, Invitrogen), 1\% p/s, 1\% ITS+ Premix, $0.04 \mathrm{mg} / \mathrm{mL}$ proline (P5607, Sigma), $0.1 \mathrm{mM} \mathrm{AsAP}$ and $10^{-7} \mathrm{M}$ dexamethasone (D1756, Sigma)) with the addition of $10 \mathrm{ng} / \mathrm{mL}$ recombinant human transforming growth factor- $\beta 1$ (TGF- $\beta 1 ; 240-B, R \& D$ Systems, Minneapolis, USA) and $100 \mathrm{ng} / \mathrm{mL}$ recombinant human bone morphogenetic protein-2 (BMP-2; 355-BM, R\&D systems). After 21 days of culture $\left(37^{\circ} \mathrm{C}, 5 \% \mathrm{CO}_{2} / 21 \% \mathrm{O}_{2}\right)$, pellets were collected for histological ( $n=$ $3 /$ donor/condition) and biochemical ( $n=3 /$ donor/condition) evaluation. Histological evaluation of glycosaminoglycan (GAG) deposition using Toluidine blue 0 (0.04\%, Sigma; Basic Blue $17,86 \%$ dye, dissolved in $0.2 \mathrm{M}$ acetate buffer), biochemical analysis of GAG release and deposition using a dimethyl methylene blue (DMMB, Sigma) assay, and determination of the DNA content using the Qubit ${ }^{\text {TM }}$ dsDNA HS Assay (Thermofisher Scientific), were performed as per methods described previously [23] .

For osteogenic and adipogenic differentiation, normal $(n=6)$ and OA $(n=6)$ P2 cSM-MSCs were seeded at a density of $\pm 1 \times 10^{3}$ (osteogenic) or $\pm 4 \times 10^{3}$ (adipogenic) cells $/ \mathrm{cm}^{2}$ in 6-well plates. Technical duplicates per donor were cultured in osteogenic differentiation medium (DMEM with 10\% FBS, $0.1 \mathrm{mM}$ AsAP, $1.25 \mathrm{\mu g} / \mathrm{mL}$ Fungizone, $10 \mathrm{mM}$-glycerophosphate (G9422, Sigma-Aldrich) and $10^{-7} \mathrm{mM}$ dexamethasone) or adipogenic differentiation medium (DMEM with 10\% FBS, $0.1 \mathrm{mM}$ AsAP, $1.25 \mu \mathrm{g} / \mathrm{mL}$ Fungizone, $10^{-6} \mathrm{mM}$ dexamethasone, $0.2 \mathrm{mM}$ indomethacin (17378, Sigma-Aldrich), $0.01 \mathrm{mg} / \mathrm{mL}$ recombinant human insulin (19278, Sigma-Aldrich), 0.5 mM 3-isobutyl-1-methylxanthine (15879, SigmaAldrich) and $5 \mu \mathrm{M}$ rosiglitazone (R2408, Sigma-Aldrich)). Negative controls received expansion medium. After 21 days, cell monolayers were fixed with 4\% NBF, and stained with Alizarin Red S staining solution (2\% Alizarin Red S (A5533, Sigma-Aldrich) for evaluation of calcium deposits or Oil Red 0 staining solution ( $0.3 \%$ Oil Red 0 (00625, Sigma-Aldrich) for evaluation of intra-cellular lipid droplets.

\section{Gene expression analysis by RT-qPCR of native SM tissue and P2 cSM-MSCs}

Snap frozen SM tissue samples wrapped in aluminium foil were reduced to powder using a hammer. After lysis of the homogenate with QIAzol Lysis Reagent (79306, Qiagen, Venlo, the Netherlands), total RNA was extracted using the RNeasy Mini Kit (74104, Qiagen) according to the manufacturer's instructions, including an on-column DNase step. Total RNA of P2 cSM-MSCs was isolated using the RNeasy Micro kit (74004, Qiagen), after lysis with RLT buffer containing $1 \mathrm{mg} / \mathrm{ml}$ 2-mercaptoethanol (M3701, Sigma-Aldrich, Saint Louis, USA). RNA quality and quantity were measured with a Bioanalyzer (Agilent RNA 6000 Nano Kit, Agilent Technologies, Amstelveen, the Netherlands). Subsequently, cDNA was produced using the iScript ${ }^{\mathrm{TM}}$ CDNA Synthesis Kit (Bio-Rad, Lunteren, the Netherlands) with a similar RNA input for all samples (SM tissue: 200 ng, P2 cSM-MSCs: 350 ng), following manufacturer's instructions. 
Quantitative RT-PCR was performed using IQ SYBR Green SuperMix and a CFX384 Touch $^{\text {TM }}$ Real-Time $^{\text {Tim }}$ PCR Detection System (Bio-Rad) according to the manufacturer's protocols. Pathways related to (synovial) inflammation, the chondrogenic and osteogenic lineage, and SM and MPC markers were investigated using canine specific primers (Table 1). Gene expression of MPC markers was included because canine specific antibodies were not available for all common MPC CD markers. Relative expression was estimated using the efficiency-corrected delta-delta $\mathrm{Ct}(\Delta \Delta \mathrm{Ct})$ method, employing 7 reference genes (Table 1). If the mean Cq value of reference genes was above 35 , the sample was excluded. 
Table 1

RT-qPCR primers. F: Forward; R: Reverse

\begin{tabular}{|c|c|c|c|}
\hline Category & Gene & Primer Sequence & $\begin{array}{l}\text { Annealing temperature } \\
\left({ }^{\circ} \mathrm{C}\right)\end{array}$ \\
\hline \multirow[t]{6}{*}{ Synovial membrane } & PRG4 & F: CCCATATACTTGCTGCTCCT & 60 \\
\hline & & R: GCATCTCTAGAATACCCTTCCC & \\
\hline & CD55 & F: GCTTCACCCTGATTGGAGAG & 60 \\
\hline & & $\begin{array}{l}\text { R: } \\
\text { CTGTAGAAGTCTGAGAACCTCTG }\end{array}$ & \\
\hline & HAS2 & F: TTGACCCTGCCTCATCTG & 59 \\
\hline & & R: AGCCATCCAGTATCTCACA & \\
\hline \multirow[t]{4}{*}{ Alarmins } & S100A8 & F: GCCATAAACTCCCTCATTGAG & 63 \\
\hline & & R: ACTCTTGGAACCAGGTGTC & \\
\hline & S100A9 & F: GAGACCATCATCAACATCTTCC & 58 \\
\hline & & R: TGATCTTGTTTATGGCGTTGTC & \\
\hline \multirow[t]{4}{*}{ MMPs } & $M M P-3$ & F: CCCAAGTGGAGGAAAACTCA & 60 \\
\hline & & R: САССТССТTCCAGACATTCAG & \\
\hline & MMP-9 & F: CGCATGACATCTTCCAGTACCA & 63 \\
\hline & & R: CCGAGAATTCACACGCCAGTA & \\
\hline \multirow{10}{*}{$\begin{array}{l}\text { Cytokines/ } \\
\text { Chemokines }\end{array}$} & $I L-1 B$ & F: TGCTGCCAAGACCTGAACCAC & 68 \\
\hline & & $\begin{array}{l}\text { R: } \\
\text { TCCAAAGCTACAATGACTGACACG }\end{array}$ & \\
\hline & $I L-6$ & $\begin{array}{l}\text { F: } \\
\text { GAGCCCACCAGGAACGAAAGAGA }\end{array}$ & 65 \\
\hline & & R: CCGGGGTAGGGAAAGCAGTAGC & \\
\hline & IL-8 & F: CTGTTGCTCTCTTGGCAGC & 63 \\
\hline & & R: GGGATGGAAAGGTGTGGAG & \\
\hline & IL-18 & F: GAGGATATGCCCGATTCTGA & 56 \\
\hline & & R: TCCGGAGGACTCATTTCTG & \\
\hline & CCL2 & F: AGCCAGATGCAATTATTTCTCC & 60 \\
\hline & & R: GACGGTCTTGAAGATCACAG & \\
\hline
\end{tabular}




\section{Category}

Gene

Primer Sequence

$\operatorname{cox} 2$

F: TTCCAGACGAGCAGGCTAAT

R: GCAGCTCTGGGTCAAACTTC

Reference genes

ran

$\begin{array}{ll}\text { RPL13 } & \\ \text { RPS5 } & \\ & \end{array}$

RPS19

HPRT

F: AGCTTGCTGGTGAAAAGGAC

R: TTATAGTCAAGGGCATATCC

F: GCCGGAAGGTTGTAGTCGT

R: GGAGGAAGGCCAGGTAATTC

F: TCACTGGTGAGAACCCCCT

R: CCTGATTCACACGGCGTAG

(2)

YWHAZ

F: ССTTCСTCAAAAAGTCTGGG

R: GTTCTCATCGTAGGGAGCAAG

$S D H A$

F: GCCTTGGATCTCTTGATGGA

R: TTCTTGGCTCTTATGCGATG

$T B P$

F: CTATTTCTTGGTGTGCATGAGG

R: CCTCGGCATTCAGTCTTTTC

F: CGAAGTTGCTGCTGGTGA

R: TTGCATTTCCTTTTTGCTGA

Category

Gene

Primer Sequence

F:

GATGCCTACAACTCCCTTTCCTCA

R: CATTTTCCCCTGTTCCATTCACC

CD34

F: TCAGGGCCCCCGACATCTC

65

$\mathrm{R}:$

TCTCTGCTCACCCCTCTGGAAAAA

CD44

F: CTTCTGCAGATCCGAACACA

60

R: GAGTAGAAGCCGTTGGATGG

$C D 73$

F: CTCCAACACATTCCTTTACAC

R: ACTCAACCTTCAAATAGCCT

$C D 90$
62

61 58

Annealing temperature

$\left({ }^{\circ} \mathrm{C}\right)$

60

58

61

62

57

Annealing temperature

$\left({ }^{\circ} \mathrm{C}\right)$

58 


\section{Category}

Gene Primer Sequence

Annealing temperature

$\left({ }^{\circ} \mathrm{C}\right)$

\begin{tabular}{|c|c|c|c|}
\hline & & $\begin{array}{l}\text { R: } \\
\text { TGGTGGTGAAGCCGGATAAGTAGA }\end{array}$ & \\
\hline & CD105 & F: CATCCTTCACCACCAAGAG & 60 \\
\hline & & R: CAGATTGCAGAAGGACGG & \\
\hline & $C D 146$ & F: GGGAATGCTGAAGGAAGG & 63 \\
\hline & & R: CTTGGTGCTGAGGTTCTG & \\
\hline & CD166 & F: AAGCGTCATAAACCAAACAG & 61 \\
\hline & & R: TATAGCAGAGACATTCAAGGAG & \\
\hline & VCAM-1 & F: CTACAAGTCTACATCTCACCCA & 58 \\
\hline & & R: TTCCAGAATCTTCCAGCCTC & \\
\hline Chondrogenic & $A C A N$ & F: GGACACTCCTTGCAATTTGAG & 61 \\
\hline & & R: GTCATTCCACTCTCССTTCTC & \\
\hline & SoX9 & F: CGCTCGCAGTACGACTACAC & 62 \\
\hline & & R: GGGGTTCATGTAGGTGAAGG & \\
\hline & $\operatorname{COL} 2 A 1$ & F: GCAGCAAGAGCAAGGAC & 65 \\
\hline & & R: TTCTGAGAGCCCTCGGT & \\
\hline & COL $1 A 1$ & F: GTGTGTACAGAACGGCCTCA & 61 \\
\hline & & R: TCGCAAATCACGTCATCG & \\
\hline Osteogenic markers & SPARC & F: TCTGTATGAAAGGGATGAGGAC & 64 \\
\hline & & R: GCTTCTCGTTCTCGTGGA & \\
\hline & RUNX2 & F: AACGATCTGAGATTTGTGGGC & 64 \\
\hline & & R: TGTGATAGGTGGCTACTTGGG & \\
\hline & $B G L A P$ & F: CTGATGGTCCTTGCCCT & 62 \\
\hline & & R: CTTGGACACGAAGGTTGC & \\
\hline & PTHR1 & F: GACCACATCCTTTGCTGG & 51 \\
\hline & & R: CAAACACCTCCCGTTCAC & \\
\hline
\end{tabular}

\section{Flow cytometry of cSM-MPCs and P2 cSM-MSCs}


Surface marker expression of normal and OA cSM-MPCs was conducted directly after digestion $(n=10$ per conditions, $\pm 0.5-1 \times 10^{6}$ cells/reaction) and P2 cSM-MSCs $\left(n=8\right.$ per condition, $\pm 1-2 \times 10^{5}$ cells/reaction). Zombie Violet ${ }^{\mathrm{TM}}$ Fixable Viability Kit (423114, BioLegend, San Diego, USA; concentration proprietary) was diluted 1:500 in flow cytometry staining buffer (00-4222-26, Invitrogen) and used at 1 $\mu \mathrm{l} / \mathrm{L} \times 10^{4}$ cells. Cells were incubated in Zombie Violet ${ }^{\mathrm{TM}}$ for 15 to 30 minutes at room temperature (RT), protected from light. Subsequently, cells were washed with $400 \mu \mathrm{L}$ flow cytometry staining buffer (004222-26, Invitrogen) and resuspended in $50 \mu \mathrm{L}$ buffer. At this point, cells were incubated with a combination of antibodies against surface markers CD90, CD44, CD73, CD271, CD34 and CD45 (Table 2) for 15 minutes at $4^{\circ} \mathrm{C}$ in the dark. One reaction per donor was left unstained as a negative control. Following antibody incubation, cells were washed with staining buffer and transferred to $5 \mathrm{~mL}$ Falcon ${ }^{\circledR}$ round-bottom polypropylene tubes (352063, Corning Life Sciences) for data acquisition. Data collection was performed with CytExpert Software (Version 2.2.0.97, Beckman Coulter, Brea, USA) on a CytoFLEX S Flow Cytometer (Beckman Coulter) and analysed using CytExpert Software version 2.2.0.97. Firstly, small debris was excluded in forward scatter (FSC)/side scatter (SSC) plots. Then, unstained reactions were used to distinguish between background and specific fluorescence signal of each antibody. Based on these gates, the relative numbers of positively or negatively stained events were determined. Dead and hematopoietic cells were excluded by negatively selecting for Zombie Violet ${ }^{\mathrm{TM}} / \mathrm{CD} 45$-stained events, and percentages of CD90, CD73, CD44, CD34 and CD271 positively stained events were evaluated within the CD45- fraction.

Table 2

Antibodies used in flow cytometry. No.: Number; PE: R-Phycoerythrin; FITC: Fluorescein isothiocyanate

\begin{tabular}{|lllllll|}
\hline Target & $\begin{array}{l}\text { Catalog } \\
\text { No. }\end{array}$ & Manufacturer & Host & Reactivity & Clone & Fluorochrome \\
\hline CD90 & $\begin{array}{l}12-5900- \\
42\end{array}$ & eBioscience & Rat & Dog & YKIX337.217 & PE \\
\hline CD73 & bs-4834R & $\begin{array}{l}\text { Bioss } \\
\text { antibodies }\end{array}$ & Rabbit & $\begin{array}{l}\text { Human, Mouse, } \\
\text { Rat, Dog, } \\
\text { Chicken }\end{array}$ & Polyclonal & FITC \\
\hline CD271 & $\begin{array}{l}12-9400- \\
42\end{array}$ & Invitrogen & Mouse & $\begin{array}{l}\text { Dog, Human, } \\
\text { Mouse }\end{array}$ & ME20.4 & PE \\
\hline CD44 & $\begin{array}{l}11-5440- \\
42\end{array}$ & Invitrogen & Rat & Dog & YKIX337.8 & FITC \\
\hline CD45 & $\begin{array}{l}48-5450- \\
42\end{array}$ & Invitrogen & Rat & Dog & YKIX716.13 & eFluor 450 \\
CD34 & FAB3346S & R\&D & Mouse & Dog & IH6 & Alexa Fluor \\
\hline
\end{tabular}

\section{Immunohistochemical evaluation}


To assess the location of cMPCs in the native SM immunohistochemical staining of the MPC markers CD90 (ab92574, Abcam), CD73 (LS-B8284, IHCPlus), CD44 (MA1-10225, ThermoFisher Scientific), CD271 (14-9400-82, eBioscience) and CD34 (bs-0646R, Bioss Antibodies) was performed (extensive protocols provided in Supplementary Table 2). For the quantification, images were captured using an Olympus BX51 microscope (Olympus, 100x magnification). Three to six random regions of interest (ROIs) were captured based on availability and analysed by a veterinary pathologist (SAK). The captured images were imported in the Image ProPlus 6.0 software (Media Cybernetics) to quantify the percentage of DABstained area.

\section{Statistical analyses}

Statistical analysis was performed using R Statistics (R version 3.6.3 [24], RStudio version 1.2.5033 [25]). Normality was tested via QQ plots, histograms, and Shapiro Wilk tests. If the data were normally distributed, linear mixed models were employed. If the data was not normally distributed, a KruskalWallis test and Dunn's Multiple Comparison Test were used. P values of the RT-qPCR analysis were subjected to corrections for multiple testing (Benjamini-Hochberg False Discovery Rate). Effect sizes (ES) with 95\% confident intervals $(\mathrm{Cl})$ were calculated using Hedge's $\mathrm{g}(\mathrm{HG})$ for normally distributed data and Cliff's delta (CD) for non-normally distributed data. Outcomes were considered relevant if $p<0.05$ or $p<$ 0.1 with a large ES. Furthermore, a difference was considered biologically relevant if the expression was undetectable in one of the groups.

\section{Results}

The synovial membrane of OA knee joints had a higher synovitis grade and increased expression of inflammation markers

The presence of synovitis in the SM derived from OA knee joints was confirmed by histology. Compared to normal SM, the total OARSI score in the OA SM was increased ( $p=0.018$, ES (HG): 1.4), defined by increased cell numbers in the synovial lining ( $p=0.0016$, ES (CD): -0.8$)$ and increased infiltration of inflammatory cells ( $p=0.0046$, ES (CD): -0.7$)$ (Figure 1A, 1B). At the transcriptional level, the synoviocyte marker CD55 was less expressed in the OA as compared to normal SM $(p<0.0001$, ES (HG): 1.8).

Furthermore, the gene expression levels of pro-inflammatory cytokines $I L-1 \beta, I L-8$ and $I L-6$, the chemokine CCL2, and the synovitis markers S100A8, and S100A9 were higher in the OA SM $(p<0.0001, \mathrm{ES}(\mathrm{HG})>$ 1.5) (Figure 1C). The synovial membrane markers Lubricin, and HAS2, and the inflammation markers $M M P 3$, and $-9, I L-18$ and COX2 did not differ between groups.

Cell culture

OA cSM-MPCs possess a lower CFU capacity

The optimal density for the CFU assay was $\pm 0.25 \times 10^{3}$ cells/petri dish for the normal cSM-MPCs and \pm $1 \times 10^{3}$ cells/petri dish for the OA cSM-MPCs (Figure 2A). A lower percentage of CFU forming cells was 
found for OA compared to normal cSM-MPCs ( $p=0.01$, ES (CD): 0.7) (Figure 2A).

Upon culture and expansion, normal and OA cSM-MSCs showed a fibroblast like morphology. After P5, population doublings declined in normal and OA cSM-MSCs (Figure 2B, Supplementary figure 2). The cumulative population doublings were lower at $\mathrm{PO}(p=0.013$, ES (HG): 1.6) and $\mathrm{P} 9(p=0.12$, ES (HG): 1.5) for OA compared to normal cSM-MSCs (Figure 2B).

Normal and OA CSM-MSCs had a similar percentage of senescent cells at all passages. The percentage of senescent cells was increased at P5 and P10 as compared to P2 for normal (P5 and P10: $p=0.0012$, $\mathrm{ES}(\mathrm{HG})>2.0$ ) and OA cSM-MPCs (P5: $p=0.02$, ES (HG): 1.2; P10: $p=0.0003$, ES (HG): 3.7) (Figure 2C). Marker expression upon expansion at $P 2$ is comparable between $O A$ and normal cSM-MSCs

Upon expansion in vitro, the CD marker expression profile of MSCs shifts towards the MSC marker expression reported by the ISCT[14, 26]. CD markers and the expression of chondrogenic and osteogenic genes were measured in P2 expanded cells. On flow-cytometry both normal and OA P2 cSM-MSCs showed a high expression ( 99\%) of CD90 and CD44, variable expression of CD73 and CD271 and undetectable expression ( $<1 \%$ ) of CD34 and CD45 (Figure 3A). At the transcriptional level, gene expression of MPC/MSC markers was detectable, but without differences (Figure 3B), and expression of chondrogenic and osteogenic markers between the cultured, undifferentiated normal and OA cSM-MSCs did not differ (Figure 3C, D).

Tri-lineage differentiation capacity

The tri-lineage differentiation potential was assessed to investigate whether the cSM-MSCs lose their multipotent differentiation potential due to the OA environment and determine their chondrogenic potential, an asset for cartilage regenerative strategies. Under chondrogenic culture conditions, at day 21 the DNA content of the OA cSM-MSC pellets was lower ( $p=0.043$, ES (HG): 1.3) compared to normal cSM-MSC pellets. 4/7 cSM-MSC donors from normal donors differentiated towards the chondrogenic lineage based on the toluidine staining for GAG deposition and the presence of chondrocyte-like cells (Figure 4A). 1/7 normal CSM-MSC donors showed COL2 immunopositivity of the extracellular matrix. cSM-MSCs of two of the OA donors showed a very mild positive toluidine blue but none of them showed COL2 immunostaining. These observations were line with the lower GAG content and GAG release in the third week of culture in the OA compared to normal cSM-MSCs ( $p=0.0054$, ES (HG): 1.4) (Figure 4B). cSM-MSC pellets from all normal and OA donors showed variable staining for COL1.

Alizarin red positive noduli, indicative for osteogenic differentiation, were found in cSM-MSCs from 4/6 normal donors, although in two donors only a few noduli were observed (Figure 5). In contrast, in cSMMSCs from all OA donors mineralized matrix was deposited with intense alizarin red staining, indicating osteogenic capacity. The negative controls were devoid of alizarin red stain.

cSM-MSCs from normal donors showed a more successful adipogenic differentiation as they had a higher number of cells with a rounded morphology and oil-red $O$ stained lipid droplets compared to the cSM-MSCs from OA donors (Figure 6). 


\section{CD marker expression in the synovial membrane}

As cSM-MSCs from normal and OA knee joints differed in their tri-lineage potential and cell culture is known to influence CD marker expression profiles [14], follow up work focused on studying the cSMMPCs in situ in the SM of normal and OA joints. For this purpose, the presence of MPC markers was investigated using RT-qPCR on snap frozen SM tissue, flow cytometry on freshly isolated cells from SM tissue digest and complemented with immunohistochemical stains of corresponding paraffin-embedded tissue. These complementary results are described below per CD marker (Figures 7 and 8 ) and in a graphical representation (Figure 9).

\section{CD90}

Within the CD45- cell population, the percentage of $C D 90+$ cells decreased in the OA compared to normal SM $(p<0.0001, E S(C D): 1.0)$ (Figure 7A). In line with this, a lower gene expression of $C D 90$ was found in the OA SM ( $p=0.08$, ES (HG): 0.7) (Figure 7B). Immunopositivity of CD90 was observed mainly in the subintimal and perivascular layer while the intimal layer was almost completely CD 90 negative, with no distinct spatial differences between the normal and the OA SM (Figure 8).

CD44

Flow cytometry demonstrated decreased percentages of CD44+ cells in the OA SM $(p=0.026, \mathrm{ES}(\mathrm{HG})$ : 0.9 ) in the CD45- cell population compared to normal (Figure 7A). On immunohistochemistry, CD44 was mainly expressed in the cell membrane of the intimal lining cells, although some expression was also variably observed in the subintimal layer and perivascular (Figure 8).

$C D 73$

There was no significant difference in the percentage of CD73+ cells in OA compared to normal SM, using flow cytometry (Figure 7A). However, gene expression of $C D 73$ was significantly lower in the OA SM ( $p=0.0046$, ES (CD): -0.7) (Figure 7B). CD73 positive cells were mostly observed in the intimal lining and perivascular layer of the synovial membrane with high variability in staining between donors for both normal and OA SM (Figure 8).

CD271

The most distinct difference between normal and OA SM was observed for CD271: in the OA SM there were less CD271+ cells ( $p<0.0001$, ES (HG): 3.0 ) compared to normal SM (Figure 7A). In addition, the double-positive, CD271+/CD44+ fraction of cells was decreased in the OA SM ( $p=0.02$, ES (CD): 1.0) (Figure 7A). Noticeable diversity in CD271 expression was observed on immunohistochemistry between OA and normal SM (Figure 8). In the OA SM, CD271 was only limited expressed in perivascular regions in the connective tissue layers adjacent to the SM. In normal SM, CD271 expression was abundant in the adipose parts of the synovial membrane and commonly observed in the intimal layer and the perivascular regions.

CD34 
Within the CD45- cell population, the CD34+ fraction was lower in the OA SM than in the normal SM ( $p<$ 0.0001 , ES (CD): 1.0) (Figure 7A). Interestingly, almost all double positive populations expressing CD34+ were lower in the OA SM compared to normal SM (CD34+/CD90+: $p<0.0001$, ES (CD): 1.0; CD34+/CD44+: $p=0.048, \mathrm{ES}(\mathrm{HG}): 1.0 ; \mathrm{CD} 34+/ \mathrm{CD} 271+: p=0.0003$, ES (CD): 1.0) (Figure 7A). This was confirmed on RT-qPCR; OA SM tissue expressed lower levels of $C D 34$ gene expression compared to normal SM ( $p<0.0001$, ES (HG): 1.8) (Figure 7B).

\section{CD146 and VCAM1}

These markers are not available as canine-specific antibody for flow cytometry and were therefore only evaluated at the transcription level. RT-qPCR showed decreased CD146 ( $p=0.024$, ES (HG): 1.0), and increased $\operatorname{VCAM1}(p=0.024$, ES (CD): 0.5) in the OA compared to normal SM (Figure 7B).

\section{Discussion}

This study aimed to isolate and characterize canine mesenchymal progenitor/stromal cells from normal and OA canine synovial membrane tissue to study the effect of the OA environment. For this purpose, surface marker expression profiling of the cMPCs in fresh SM digest and of the cultured P2 cells (referred to as cMSCs) and tri-lineage differentiation assays of cSM-MSCs were conducted. This is the first time that the tri-lineage differentiation capacity of cSM-MSCs is directly compared between OA and normal joints. The isolated cMPCs and cMSCs from normal and OA SM possessed many of the characteristics that are described for MSC by the International Society for Cellular Therapy (ISCT) [26]; a fibroblast-like morphology on culture plastic, CFU capacity, positivity for CD90, CD73 and CD44 without expression of CD45 and CD34, and tri-lineage differentiation capacity. However, distinct functional differences were observed, depending on the health state of the tissue they were derived from, being more distinct at the CD marker level in the CSM-MPCs rather than the cultured P2 CSM-MSCs. As the CD marker expression is known to change during culture[14,27], the absence of distinct differences in the $\mathrm{P} 2$ is most probably due to effects of the expansion procedure. CSM-MSCs have been described to outperform other MSC tissue sources like bone marrow and adipose tissue in chondrogenic capacity $[19,21]$. Nonetheless, the cSMMSCs from OA joints displayed lower chondrogenic and adipogenic, but better osteogenic capacity compared to cSM-MSCs from normal joints. This coincides with the findings of a direct comparison between pre-OA and OA human SM-MSCs [11] in which OA SM-MSCs possessed lower CFU capacity, a lower proliferation rate, a higher percentage of senescent cells, and lower chondrogenic differentiation capacity. Moreover, this impaired chondrogenic capacity of SM-MSCs was also reported in sheep, in which the inflammatory joint environment, created by transecting the anterior cruciate ligament, was related to a decreased cartilage pellet size and GAG deposition [28]. Furthermore, the catabolic factors in conditioned medium derived from the OA synovial membrane inhibited the chondrogenic differentiation of human MSCs in vitro [29, 30]. Altogether, this implies that the OA joint environment has a negative impact on the chondrogenic capacity of joint MPCs and MSCs generated upon MPC expansion. 
Interestingly, cSM-MSCs derived from the OA joints had a better osteogenic capacity than cMSCs from normal joints. This is in line with the increased osteogenic capacity of cSM-MSCs derived from OA joints with cruciate ligament disease compared to cSM-MSCs from OA joints with medial patella luxation[20]. The former $\mathrm{OA}$ joints typically have more severe synovial membrane inflammation while the latter come with only mild synovitis [20]. This contrasts with earlier studies, which reported no difference in osteogenic capacity between normal and OA human bone marrow- [10] and ovine SM-MSCs [31]. However, the effect of inflammatory cytokines on the osteogenic differentiation of MSCs is ambiguous. For example, TNFa has been reported to inhibit as well as promote osteoblastogenesis, depending on the cell type, the animal model, and the timing, duration and dosage of TNFa administration [32, 33]. While we cannot exclude that this contradiction could also be species-dependent, the available literature implies that the levels of inflammation within the OA environment may differentially prime the heterogenous MPC population and affect their osteogenic capacity.

The decreased CFU capacity and decreased chondrogenic differentiation capacity of cSM-MSCs from OA joints, raise the question whether the number of multipotent progenitor cells is exhausted during $O A$, and/or if their phenotype is altered priming cells towards the osteogenic lineage. Exhaustion and dysfunction of the progenitor cell population has been described for other musculoskeletal tissues with ageing and degeneration, e.g., the intervertebral disc and in fracture bone healing [34, 35]. Additionally, the OA environment might also accelerate the aging of the SM-MPCs, resulting in a decreased proliferation capacity and increased senescence [36]. Although the exact relationship between senescence and OA is still largely unknown, it is thought that cellular senescence may play a significant role in the pathology of $O A$ [37]. In turn, ageing and senescence have a negative effect on the regenerative capacities of MSCs [38]. In the present study OA cSM-MSCs had a lower population doubling in P9 compared to normal cSMMSCs, but senescence levels did not differ from normal, which is in contrast with earlier studies reporting increased senescence in OA SM-MSCs [11]. In light of the differences in senescence of bone marrowderived MSCs observed between different dog breeds [39] and the large variation observed within this study in senescence, a larger sample size is needed to determine the cofounding role of OA severity in cellular senescence of the cSM-MSCs.

Differences between studies relating to differences in species, OA severity, joint location and injury type could influence the effect of OA on MPCs in the synovial membrane. Earlier studies used immunostainings to investigate MSC/MPC markers to quantify the presence and spatial distribution of progenitor cells during $O A$ in the synovial membrane. In contrast to this study, they demonstrated an increase in CD90, CD44 and CD271 positive cells in the OA compared to normal synovium [12, 13, 40], suggesting that MPCs increase in numbers in the OA synovium. There are, however, important differences to consider while interpreting these observations. Firstly, immunostainings of tissue sections are not as quantitative as flow cytometry of an entire synovial membrane tissue sample. Secondly, CD90 was reported to be expressed in both the intimal and subintimal lining $[12,13]$, in contrast to the present study in which CD90 was mainly expressed in the lining of the canine synovial membrane. This might be a species-dependent difference; in immunostainings of human OA SM tissues with the CD90 antibody used in the present study we observed less intense staining and a CD90 perivascular localisation 
(Supplementary figure 3). Finally, OA severity influences CD marker expression: Del Rey et. al. (2016) reported that while CD271 positivity was not increased in pre-OA samples, with a moderate to severe synovitis, compared to normal synovial tissue, it was increased in OA samples [40]. In line with this, CD271 immunopositivity in our study was highest in one of the dogs with severe OA. Additionally, the joint location (hip versus knee), and injury type might also have an effect. For example, human SM-MSCs derived from the hip joints of patients with femoroacetabular impingement syndrome showed higher proliferative and chondrogenic capacity compared to SM-MSCs derived from patients with hip OA [41]. Furthermore, Wijekoon et. al. (2017) showed differences between OA SM-MSCs from dogs with medial patellar luxation and cranial cruciate ligament disease [20].

The use of MSC/MPC markers to determine the relative numbers of progenitor cells in vivo is hampered by the lack of specific markers. While the use of functional markers, such as iododeoxyuridine (IdU) to mark slow-cycling cells [3], or lineage tracing, using important developmental markers such as Gdf5[4], are promising techniques, they are not feasible to investigate progenitor cells in humans and large animal models, such as the dog. Therefore, the combination of the known MSC/MPC markers with new or less known markers is necessary in the canine species. In this study, CD34 was used as a marker for progenitor cells and was shown to decrease together with CD90 and CD44. While this marker is often considered a negative marker for MSCs [26], it is a common misconception that all CD34+ cells are hematopoietic [42], and this is presumed because isolated CD34+ MPCs lose their CD34+ positivity upon in vitro culture [42]. As such, CD34 might be an interesting MPC marker to use, although the tissue source should be considered in this context. To date, CD34 is mostly associated with adipose tissue derived MPCs [42]. In this study, the normal canine synovial membrane contained relatively more adipose tissue compared to the OA synovial membrane. Therefore, this may explain the relatively smaller fraction of CD34+ cells detected in OA samples and the decreased adipogenic capacity of the OA cSM-MSCs in this study. New markers seem to be necessary to move forward in the MPC marker field and unbiased methods such as single cell RNA sequencing of the synovium tissue digest could be used to study the heterogenous cell population in vivo identity of the SM-MPCs and discover new markers.

A major limitation of the study is that the OA donor group was significantly older compared to the normal group. Normal tissues were collected from experimental dogs euthanized in unrelated experiments that typically employ young adults. Age is known to influence MSC characteristics [43], and although no difference in senescence was found between the two groups, it would be preferable to have an older control group in which OA is excluded. However, this is a challenge, as up to $80 \%$ of the dogs older than 8 years, has radiographic or clinical signs of OA [44].

\section{Conclusion}

To exploit the regenerative capacity of synovial membrane derived progenitor cells, more in depth knowledge is needed about their role in the normal joint homeostasis and OA. This study showed that the OA environment has a negative effect on the regenerative capacity of cSM-MSCs indicated by the decreased CFU and population doublings, and decreased chondrogenic but enhanced osteogenic potency 
compared to normal cSM-MSCs. Furthermore, in the OA synovial membrane the MSC/MPC markers CD90, CD44, CD73 and CD271 were decreased, indicating a loss of MSC/MPC phenotype or a depletion of progenitor cells in the synovial membrane. These observations impact the application of SM-MSCs in cell-based treatments of OA in multiple ways. Firstly, the use of autologous SM-MSCs from the OA joint should be critically considered, as their regenerative capacity is inferior to normal SM-MSCs. Secondly, in case of exogenous MSC implantation, the OA environment may inhibit the regenerative capacity of the implanted MSCs. Considering that the effects of the OA environment are still present on CSM-MSCs in vitro at least until passage 2 , the in vitro culture of these cells could serve as model to develop new methods and test the developed strategies in improving the performance of MSCs as a cell-based treatment strategy.

\section{Abbreviations}

MPC: Mesenchymal progenitor cell

MSC: Mesenchymal stem/stromal cell

SM: Synovial membrane

OA: Osteoarthritis

RT-qPCR: Reverse transcriptase quantitative polymerase chain reaction

HE: Haematoxylin Eosin

OARSI: Osteoarthritis Research Society International

FBS: Fetal bovine serum

CFU: Colony forming unit

AsAP: Ascorbic acid 2-phosphate

bFGF: basic fibroblast growth factor

P: Passage

PD: Population doublings

NBF: Neutral buffered formalin

GAG: Glycosaminoglycan

DMMB: Dimethyl methylene blue

RT: Room temperature 
FSC: Forwards scatter

SSC: Side scatter

ROI: Region of interest

ES: Effect size

HG: Hedge's G (effect size)

CD: Cliff's delta (effect size)

ISCT: International Society for Cellular Therapy

COL: Collagen

\section{Declarations}

\section{Ethics approval and consent to participate}

Synovial membrane was collected from dogs euthanized in unrelated experiments conducted in accordance with the guidelines set by the National Central Committee for Animal Experiments (AVD \#115002016531). Synovial membrane from osteoarthritic knee joints was collected, with the owner's consent, during standard-of-care surgery.

Consent for publication

Not applicable

\section{Availability of data and materials}

All data generated or analysed during this study are included in this published article in the form of graphs. Additional datasets used and/or analysed during the current study are available from the corresponding author on reasonable request.

Competing interests

The authors declare that they have no competing interests

\section{Funding}

This project was financially supported by the Dutch Arthritis Society (LLP9 and LLP22) and NWO Applied and Engineering Sciences (P15-23). There is no further involvement in the present work of the abovementioned sources. 
BPM, SCM and MAT obtained the funding for this study. MT, NA, SCM and MAT contributed to the conception and design of the study. MT, NA, LS, SAK, BPM, and KS contributed to the experimental procedures, and collection and assembly of the data. MT, NA, LS, RN, SAK, GJVM, SCM, KS, and MAT contributed to the analysis and interpretation of the data. All authors contributed to drafting of the article and helped revising it critically for intellectual content. All authors approved the final version of this article for submission.

\section{Acknowledgements}

Not applicable.

\section{References}

1. Sacchetti B, Funari A, Remoli C, Giannicola G, Kogler G, Liedtke S, et al. No identical "Mesenchymal Stem Cells" at different times and sites: human committed progenitors of distinct origin and differentiation potential are incorporated as adventitial cells in microvessels. Stem Cell Reports 2016;6:897-913.

2. de Bari C, Dell'Accio F, Tylzanowski P, Luyten FP. Multipotent mesenchymal stem cells from adult human synovial membrane. Arthritis and Rheumatism 2001;44:1928-42.

3. Kurth TB, Dell'Accio F, Crouch V, Augello A, Sharpe PT, de Bari C. Functional mesenchymal stem cell niches in adult mouse knee joint synovium in vivo. Arthritis and Rheumatism 2011;63:1289-300.

4. Roelofs AJ, Zupan J, Riemen AHK, Kania K, Ansboro S, White N, et al. Joint morphogenetic cells in the adult mammalian synovium. Nature Communications 2017;8:1-14.

5. de Bari C, Roelofs AJ. Stem cell-based therapeutic strategies for cartilage defects and osteoarthritis. Current Opinion in Pharmacology 2018;40:74-80.

6. de Bari $C$ de, Kurth TB, Augello A. Mesenchymal stem cells from development to postnatal joint homeostasis, aging, and disease. Birth Defects Research Part C: Embryo Today: Reviews 2010;90:257-71.

7. van den Bosch MHJ, van Lent PLEM, van der Kraan PM. Identifying effector molecules, cells, and cytokines of innate immunity in OA. Osteoarthritis and Cartilage 2020;28:532-43.

8. McGonagle D, Baboolal TG, Jones E. Native joint-resident mesenchymal stem cells for cartilage repair in osteoarthritis. Nature Reviews Rheumatology 2017;13:719-30.

9. Scotti C, Gobbi A, Karnatzikos G, Martin I, Shimomura K, Lane JG, et al. Cartilage repair in the inflamed joint: considerations for biological augmentation toward tissue regeneration. Tissue Engineering - Part B: Reviews 2016;22:149-59.

10. Murphy JM, Dixon K, Beck S, Fabian D, Feldman A, Barry F. Reduced chondrogenic and adipogenic activity of mesenchymal stem cells from patients with advanced osteoarthritis. Arthritis and Rheumatism 2002;46:704-13. 
11. Huang J, Chen C, Liang C, Luo P, Xia G, Zhang L, et al. Dysregulation of the Wnt signaling pathway and synovial stem cell dysfunction in osteoarthritis development. Stem Cells and Development 2020;29:401-13.

12. Hermida-Gómez T, Fuentes-Boquete I, Gimeno-Longas MJ, Muiños-López E, Díaz-Prado S, de Toro FJ, et al. Quantification of cells expressing mesenchymal stem cell markers in healthy and osteoarthritic synovial membranes. Journal of Rheumatology 2011;38:339-49.

13. O’Brien K, Tailor P, Leonard C, Difrancesco LM, Hart DA, Matyas JR, et al. Enumeration and localization of mesenchymal progenitor cells and macrophages in synovium from normal individuals and patients with pre-osteoarthritis or clinically diagnosed osteoarthritis. International Journal of Molecular Sciences 2017;18:774.

14. Sivasubramaniyan K, Koevoet WJLM, Hakimiyan AA, Sande M, Farrell E, Hoogduijn MJ, et al. Cellsurface markers identify tissue resident multipotential stem/stromal cell subsets in synovial intimal and sub-intimal compartments with distinct chondrogenic properties. Osteoarthritis and Cartilage 2019;27:1831-40.

15. McCoy AM. Animal Models of Osteoarthritis. Veterinary Pathology 2015;52:803-18.

16. Cook JL, Kuroki K, Visco D, Pelletier JP, Schulz L, Lafeber FPJG. The OARSI histopathology initiative recommendations for histological assessments of osteoarthritis in the dog. Osteoarthritis and Cartilage 2010;18:S66-79.

17. Meeson RL, Todhunter RJ, Blunn G, Nuki G, Pitsillides AA. Spontaneous dog osteoarthritis - a One Medicine vision. Nature Reviews Rheumatology 2019;15:273-87.

18. Devireddy LR, Boxer L, Myers MJ, Skasko M, Screven R. Questions and challenges in the development of Mesenchymal Stromal/Stem Cell-based therapies in veterinary medicine. Tissue Engineering Part B, Reviews 2017;23:462-70.

19. Sasaki A, Mizuno M, Ozeki N, Katano H, Otabe K, Tsuji K, et al. Canine mesenchymal stem cells from synovium have a higher chondrogenic potential than those from infrapatellar fat pad, adipose tissue, and bone marrow. PLoS One 2018;13:e0202922.

20. Wijekoon HMS, Toyota K, Kim S, Fang J, Bwalya EC, Hosoya K, et al. Differentiation potential of synoviocytes derived from joints with cranial cruciate ligament rupture and medial patella luxation in dogs. Research in Veterinary Science 2017;114:370-7.

21. Bearden RN, Huggins SS, Cummings KJ, Smith R, Gregory CA, Saunders WB. In-vitro characterization of canine multipotent stromal cells isolated from synovium, bone marrow, and adipose tissue: A donor-matched comparative study. Stem Cell Research and Therapy 2017;8:1-22.

22. Lozano-Gerona J, García-Otín ÁL. ImageJ-based semiautomatic method to analyze senescence in cell culture. Analytical Biochemistry 2018;543:30-2.

23. Teunissen M, Verseijden F, Riemers FM, van Osch GJVM, Tryfonidou MA. The lower in vitro chondrogenic potential of canine adipose tissue-derived mesenchymal stromal cells (MSC) compared to bone marrow-derived MSC is not improved by BMP-2 or BMP-6. Veterinary Journal 2021;269:105605. 
24. R Core Team (2020). R: A language and environment for statistical computing. 2020.

25. Rstudio Team. RStudio: Integrated Development for R. 2020. https://doi.org/10.1145/3132847.3132886.

26. Dominici M, le Blanc K, Mueller I, Slaper-Cortenbach I, Marini FC, Krause DS, et al. Minimal criteria for defining multipotent mesenchymal stromal cells. The International Society for Cellular Therapy position statement. Cytotherapy 2006;8:315-7.

27. Harting MT, Jimenez F, Pati S, Baumgartner J, Cox CS. Immunophenotype characterization of rat mesenchymal stromal cells. Cytotherapy 2008;10.

28. Niemeyer P, Fechner K, Milz S, Richter W, Suedkamp NP, Mehlhorn AT, et al. Comparison of mesenchymal stem cells from bone marrow and adipose tissue for bone regeneration in a critical size defect of the sheep tibia and the influence of platelet-rich plasma. Biomaterials 2010;31:35729.

29. Heldens GTH, Davidson ENB, Vitters EL, Schreurs BW, Piek E, van den Berg WB, et al. Catabolic factors and osteoarthritis-conditioned medium inhibit chondrogenesis of human Mesenchymal Stem Cells. Tissue Engineering Part A 2012;18:45-54.

30. Fahy N, de Vries-van Melle ML, Lehmann J, Wei W, Grotenhuis N, Farrell E, et al. Human osteoarthritic synovium impacts chondrogenic differentiation of mesenchymal stem cells via macrophage polarisation state. Osteoarthritis and Cartilage 2014;22:1167-75.

31. Ando W, Heard BJ, Chung M, Nakamura N, Frank CB, Hart DA. Ovine synovial membrane-derived mesenchymal progenitor cells retain the phenotype of the original tissue that was exposed to in-vivo inflammation: evidence for a suppressed chondrogenic differentiation potential of the cells. Inflammation Research 2012;61:599-608.

32. Kotake S, Nanke Y. Effect of TNFa on osteoblastogenesis from mesenchymal stem cells. Biochimica et Biophysica Acta (BBA) - General Subjects 2014;1840:1209-13.

33. Vallés G, Bensiamar F, Maestro-Paramio L, García-Rey E, Vilaboa N, Saldaña L. Influence of inflammatory conditions provided by macrophages on osteogenic ability of mesenchymal stem cells. Stem Cell Research \& Therapy 2020;11:1-15.

34. Wagner D, Karnik S, Gunderson Z, Nielsen J, Fennimore A, Promer H, et al. Dysfunctional stem and progenitor cells impair fracture healing with age. World Journal of Stem Cells 2019;11:281-96.

35. Lyu F-J, Cheung KM, Zheng Z, Wang H, Sakai D, Leung VY. IVD progenitor cells: a new horizon for understanding disc homeostasis and repair. Nature Reviews Rheumatology 2019;15:102-12.

36. Rahmati M, Nalesso G, Mobasheri A, Mozafari M. Aging and osteoarthritis: Central role of the extracellular matrix. Ageing Research Reviews 2017;40:20-30.

37. McCulloch K, Litherland GJ, Rai TS. Cellular senescence in osteoarthritis pathology. Aging Cell 2017;16:210-8.

38. Zupan J, Drobnič M, Stražar K. Synovium-Derived Mesenchymal Stem/Stromal Cells and their promise for cartilage regeneration. Advances in Experimental Medicine and Biology 2019;1212:87106. 
39. Bertolo A, Steffen F, Malonzo-Marty C, Stoyanov J. Canine mesenchymal stem cell potential and the importance of dog breed: Implication for cell-based therapies. Cell Transplantation 2015;24:196980.

40. del Rey MJ, Faré R, Usategui A, Cañete JD, Bravo B, Galindo M, et al. CD271+ stromal cells expand in arthritic synovium and exhibit a proinflammatory phenotype. Arthritis Research \& Therapy 2016;18:1-8.

41. Murata $Y$, Uchida $S$, Utsunomiya $H$, Hatakeyama A, Nakashima $H$, Mori T, et al. Differentiation potential of Synovial Mesenchymal Stem Cells isolated from hip joints affected by femoroacetabular impingement syndrome versus osteoarthritis. Arthroscopy: The Journal of Arthroscopic \& Related Surgery 2020;36:2122-33.

42. Sidney LE, Branch MJ, Dunphy SE, Dua HS, Hopkinson A. Concise review: Evidence for CD34 as a common marker for diverse progenitors. Stem Cells 2014;32:1380-9.

43. Stolzing A, Jones E, McGonagle D, Scutt A. Age-related changes in human bone marrow-derived mesenchymal stem cells: Consequences for cell therapies. Mechanisms of Ageing and Development 2008;129:163-73.

44. Johnston SA. Osteoarthritis: joint anatomy, physiology, and pathobiology. Veterinary Clinics of North America: Small Animal Practice 1997;27:699-723.

\section{Figures}


A)

Normal

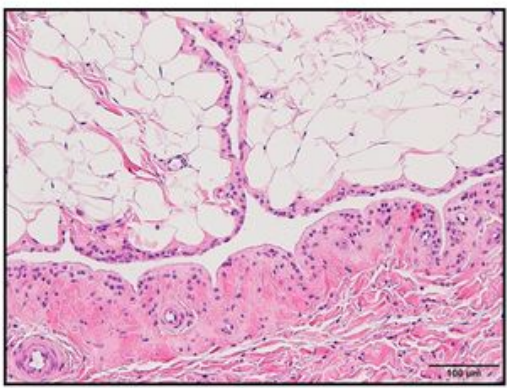

$\mathrm{OA}$

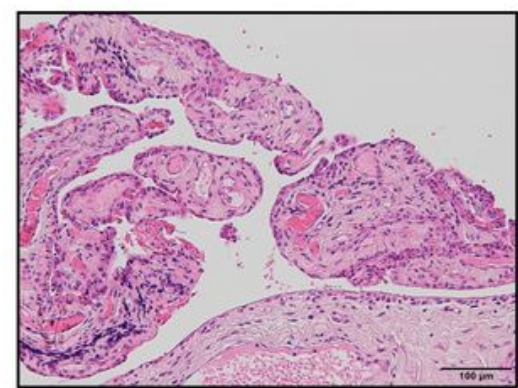

B) OARSI score Synovial Membrane

Total OARSI score

Lining Cells

Cellular Infiltration
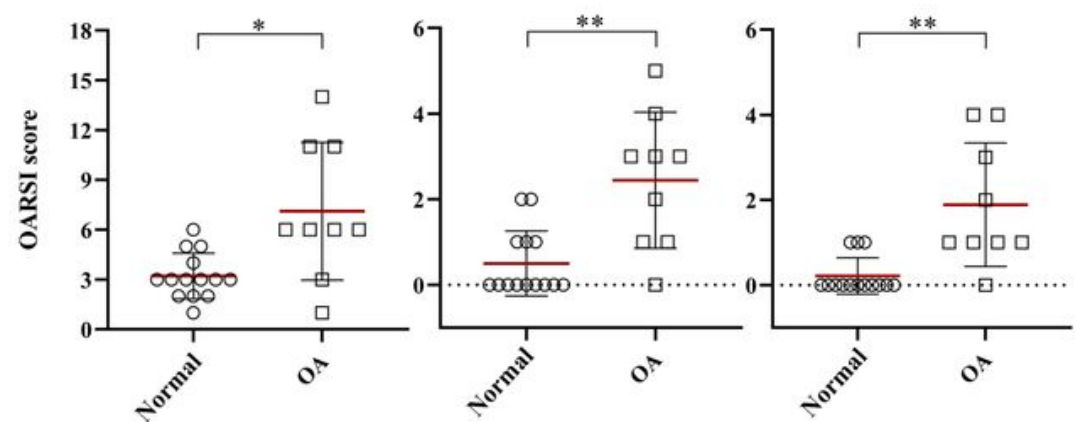

C)

RT-qPCR Synovial Membrane
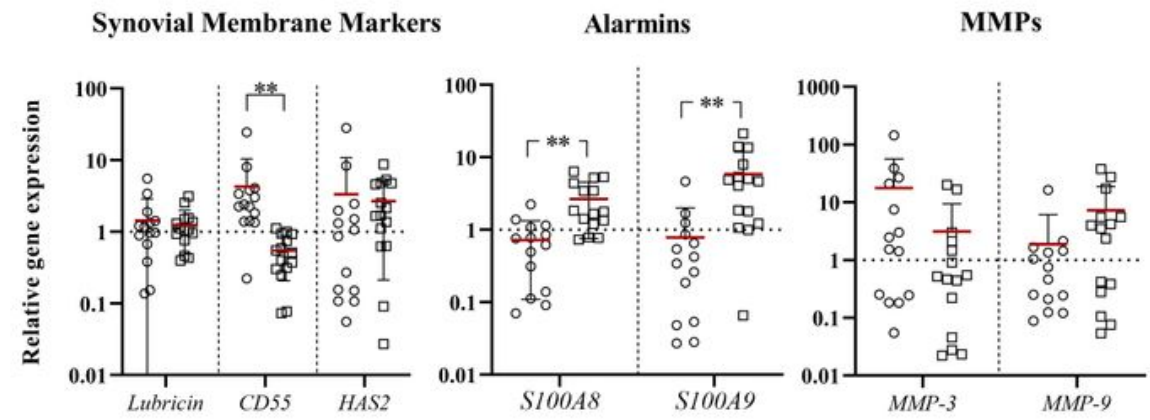

Cytokines and Chemokines

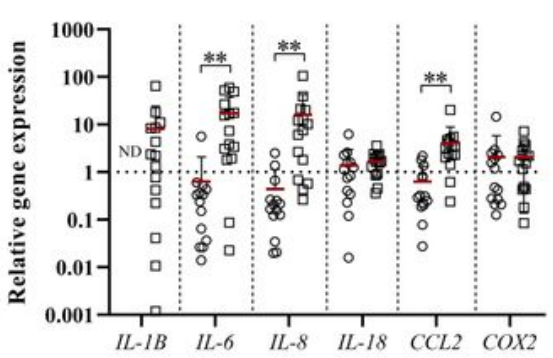

- Normal

口 $\mathrm{OA}$

\section{Figure 1}

Assessment of inflammation in the synovial membrane. (A) Representative images of normal and osteoarthritic (OA) synovial membrane stained with hematoxylin/eosin. The scale bar is set at $100 \mu \mathrm{M}$. (B) OARSI scoring of normal (circle) and OA (square) synovial membrane. The total OARSI score consists of the sum of the individual categories; lining cell characteristics, lining characteristics (not shown), and the evaluation of cellular infiltration. (C) RT-qPCR analysis of markers of the synovial membrane and 
synovitis. Gene expression is shown on a log scale as the relative gene expression compared to the mean of all samples within a gene. IL-1 $\beta$ was not detected (ND) in the normal samples. Each dot represents an individual donor. ${ }^{*} p<0.05, * * p<0.01$.

\section{Figure 2}

Cell culture characteristics. (A) Colony forming unit (CFU) potential. The optimal seeding cell density was determined for normal (circle) and osteoarthritic (OA, square) donors by testing three densities. For OA donors, a cell density of $1.0^{\star} 10^{3}$ was chosen, and for the normal donors a cell density of $0.25^{\star} 10^{3}$. The amount of CFUs was displayed as the percentage of CFU of the total seeded cells. (B) Population doublings are displayed as the cumulative population doublings per passage for normal (black dots) and OA (clear dots). (C) Senescence of the normal and OA cSM-MSCs was investigated using a $\beta$ galactosidase assay in passage $(P) 2,5$, and 10 . The number of senescent cells (stained blue in the microscopic images (black arrows)) was depicted as the percentage of senescent cells of the total cells. Each dot represents an individual donor. $\# p<0.15$ with a large effect size $* p<0.05,{ }^{\star *} p<0.01$.

\section{Figure 3}

Marker expression in passage 2 (P2) cultured CSM-MSCs. (A) Evaluation of CD markers by flow cytometry in P2 cultured normal (circle) and OA (square) cSM-MSCs. Expression is shown as the percentage (\%) of positive cells (events) of all live cells for CD90, CD44, CD73, CD271, CD34 and CD45. RT-qPCR analysis of (B) CD marker expression, (C) chondrogenic lineage and (D) osteogenic lineage markers in P2 cultured CSM-MSCs. Gene expression of the markers is shown on a log scale as the relative gene expression compared to the mean of all samples within a gene. Each dot represents an individual donor.

\section{Figure 4}

Chondrogenic differentiation of normal and osteoarthritic (OA) synovial membrane-derived cMSCs. (A) Evaluation of glycosaminoglycan (GAG) deposition and collagen content. $5 \mu \mathrm{m}$ sections of pellets cultured for 21 weeks in chondrogenic differentiation medium containing $10 \mathrm{ng} / \mathrm{ml} \mathrm{TGF-} \beta 1$ and 100 $\mathrm{ng} / \mathrm{ml} \mathrm{BMP-2}$, were stained with toluidine blue (scale bar $=100 \mu \mathrm{M})$. A representative image of every donor is shown. One donor $(X)$ was lost during processing. Additionally, immunohistochemical analysis of collagen (COL) content was performed for COL type I and II using DAB (3, 3'-diaminobenzidine, orange/brown staining) (scale bar $=200 \mu \mathrm{m})$. Biochemical evaluation of the GAG (B) and DNA (C) content in $\mu \mathrm{g}$. Each dot represents an individual donor. ${ }^{*} p<0.05,{ }^{* *} p<0.01$. 


\section{Figure 5}

Evaluation of osteogenic differentiation by Alizarin-red staining. Calcium (stained in red) and the presence of noduli are representative of successful osteogenic differentiation. Representative images of every donor were obtained using the brightfield setting of an Olympus IX51 inverted microscope. The negative controls received expansion medium for 21 days. The scale bar is set at $500 \mu \mathrm{m}$.

\section{Figure 6}

Evaluation of adipogenic differentiation by Oil-red-0 staining. Lipid droplets (stained in red) and the more rounded cell morphology are representative of successful adipogenic differentiation. Representative images of every donor were obtained using the phase contrast setting of an Olympus IX51 inverted microscope. The negative controls received expansion medium for 21 days. The scale bar is set at 100 $\mu \mathrm{m}$.

\section{Figure 7}

Quantitative evaluation of CD marker expression in the synovial membrane. (A) Evaluation of CD markers by flow cytometry. Expression is shown as the percentage of positive cells (events) within the CD45 negative cell population for each single marker and for the co-expression of markers for normal (circle) and osteoarthritic (OA, square) synovial membrane. (B) RT-qPCR analysis of CD marker expression in the synovial membrane. Gene expression of the CD markers is shown on a log scale as the relative gene expression compared to the mean of all samples within a gene. Each dot represents an individual donor. * $p<0.05,{ }^{* *} p<0.01$.

\section{Figure 8}

Evaluation of the CD marker location in the synovial membrane. Immunohistochemical analysis using DAB (3, 3'-diaminobenzidine, orange/brown staining) was performed to identify the location of CD90, CD44, CD73, CD271, and CD34 expression in the normal (circle) and osteoarthritic (OA, square) synovial membrane. Representative images, containing both fibrous and adipose type synovial membrane, were chosen. The scale bar is set at $50 \mu \mathrm{m}$. Quantification of the DAB staining was performed with Image ProPlus 6.0 software (Media Cybernetics) in 3 to 6 random regions of interest (ROI) per donor, resulting in a mean percentage of DAB positive staining ares for each donor. 


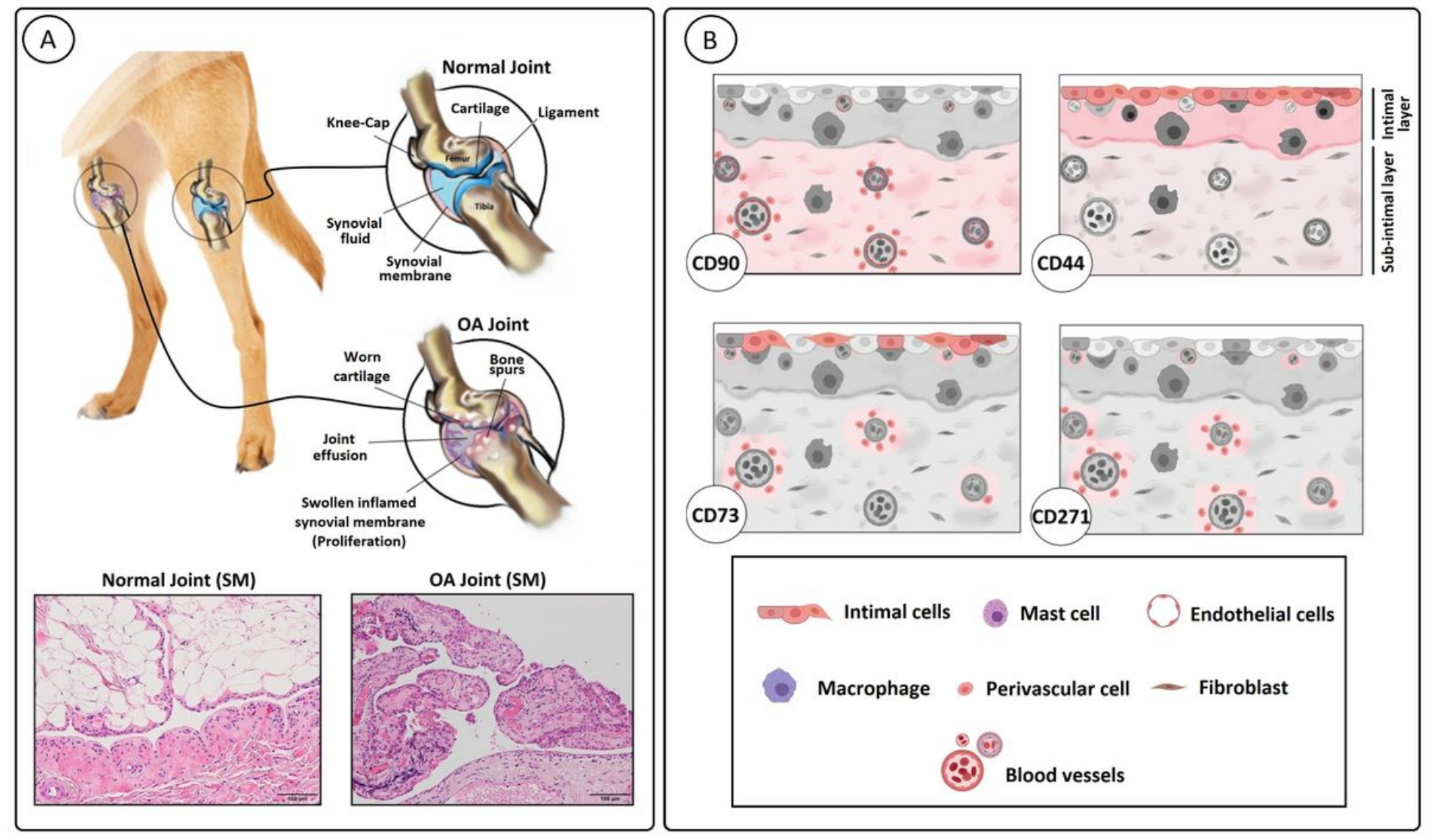

\section{Figure 9}

Graphical overview of the CD marker expression in the synovial membrane. (A) Synovial membrane (SM) was collected from normal and OA knee joints. (B) Immunohistochemical staining was performed for CD90, CD44, CD73 and CD271. Tissue expression of these markers is visualized in red. Immunopositivity of CD90 was observed mainly in the subintimal and perivascular layer. CD44 was mainly expressed in the intimal lining cells, although variable expression was observed in the subintimal layer and perivascular. CD73 positive cells were mostly observed in the intimal lining and perivascular layer. CD271 was expressed perivascular and in the normal SM also in the intimal layer of the adipose parts of the synovial membrane.

\section{Supplementary Files}

This is a list of supplementary files associated with this preprint. Click to download.

- SupplementaryMaterialMTeunissen.docx 